\title{
Pacific
}

Journal of

Mathematics

\section{GEOMETRY OF TRINOMIALS}

Aaron MELMAN

Volume 259 No. 1

September 2012 


\title{
GEOMETRY OF TRINOMIALS
}

\author{
AARON MELMAN
}

\begin{abstract}
The location of the zeros of a general trinomial was analyzed in the late 19th and early 20th centuries. We reexamine this problem and obtain new zero inclusion regions in the complex plane that complement and improve known results. Our main contribution is the derivation of smaller annular sectors containing the zeros of a trinomial that take into account the magnitude of the coefficients, which is unlike existing results where such sectors are rigid.
\end{abstract}

\section{Introduction}

Locating the zeros of a trinomial of the form $p(w)=a_{n} w^{n}+a_{k} w^{k}+a_{0}$ (where $w, a_{0}, a_{k}, a_{n} \in \mathbb{C}$ satisfy $a_{0} a_{k} a_{n} \neq 0$, and $n, k$ are positive integers such that $n \geq 3$ and $1 \leq k \leq n-1)$ is a problem with a long history that was studied by several authors, such as Biernacki [1928], Herglotz [1922], Kempner [1922], Landau [1906; 1907], and Nekrassoff [1887], some of whom were also mentioned in [Marden 1966, pp. 80, 147, 165]. Their results take the form of bounds on the magnitude of the zeros or of sectors in the complex plane containing the zeros. The oldest of these works, [Nekrassoff 1887] — based on an earlier paper by that same author from 1883 - was published only 25 years after Rouché's theorem [1862]; it is interesting that, although mentioned, this theorem was not used there.

The Hungarian mathematician Jenő Egerváry, whose work on the location of trinomial zeros was summarized in [Szabó 2010], used an interpretation of the zeros as the equilibrium points of a force field to obtain annular sectors in the complex plane containing the zeros. However, the radii of the annuli are themselves the moduli of the zeros of related trinomials, making this more a theoretical result.

Let us now use [Nekrassoff 1887] as a convenient reference to explain what we intend to do here since, in addition to its own unique results, it also reflects the results from the other references (including later ones) when applied to a trinomial. We first simplify the aforementioned trinomial by dividing $p(w)$ by $-a_{0}$ and setting $w=\left(-a_{0} / a_{n}\right)^{1 / n} z$ for any $n$-th root, which transforms $p(w)$ into $q(z)$,

MSC2010: 12D10, 15A18, 30C15.

Keywords: Rouché, trinomial, zero, root, location, bound, estimate. 
where

$$
q(z)=z^{n}-\left(\frac{a_{k}}{a_{0}}\right)\left(-\frac{a_{0}}{a_{n}}\right)^{k / n} z^{k}-1 .
$$

In addition, we will assume that $\operatorname{gcd}(k, n)=1$, so that the trinomial cannot be simplified by a substitution of the form $y=z^{\ell}$. From here on, we will investigate the location of the zeros of

$$
q(z)=z^{n}-a z^{k}-1
$$

where

$$
a=\left(\frac{a_{k}}{a_{0}}\right)\left(-\frac{a_{0}}{a_{n}}\right)^{k / n}
$$

The zeros of $p(w)$ can then easily be recovered from those of $q(z)$.

In Satz I and Satz II (Theorem I and Theorem II) of [Nekrassoff 1887], open annular sectors are derived, each containing a zero of the polynomial $q: n$ contiguous ones in Satz I and two groups of $k$ and $n-k$ contiguous ones in Satz II. Satz I applies when the modulus of the coefficient $a$ is below a certain threshold, while Satz II applies for values above that threshold. However, these annular sectors (as is true for all the other aforementioned references) depend only on $n$ and/or $k$, and the insensitivity to the coefficient $a$ makes the angles they subtend unnecessarily large in general. Just one example of an obvious shortcoming is that such rigid annular sectors cannot reflect the fact that the zeros of $q$ converge to the $n$ roots of unity as $a \rightarrow 0$.

We will qualitatively improve Satz I and Satz II of [Nekrassoff 1887] and similar results in the other previously mentioned references by deriving smaller disjoint annular sectors each containing a zero of $q$ that are easy to obtain and that are, unlike previous results, sensitive to the modulus of the coefficient $a$. The angles they subtend tend to zero as $|a| \rightarrow 0$ and as $|a| \rightarrow+\infty$. These results are stated in Theorem 4.1 and Theorem 5.3.

In Section 2 we collect a few definitions and lemmas to be used in subsequent sections. In Section 3 we derive an inclusion region for the zeros that is valid for any value of $|a|$, whereas disjoint inclusion regions for the smallest $k$ and largest $n-k$ zeros are derived in Section 4 when $|a|$ exceeds a well-defined value, and disjoint inclusion regions for all the zeros are derived in Section 5 when $|a|$ falls below another well-defined value. All inclusion regions are illustrated by examples. An Appendix deals with some numerical issues, which are not the focus of this work.

\section{Preliminaries}

In this section we state several definitions as well as a number of lemmas so that we may easily refer to them later on. Throughout we will denote by $O(c ; \rho)$ an 
open disk centered at $c$ with radius $\rho$ and its closure by $\bar{O}(c ; \rho)$. The complement of a set $H$ is denoted by $H^{c}$.

For convenience, since we refer to it often, we begin by stating Rouché's wellknown theorem.

Theorem 2.1 ([Rouché 1862]; see also [Lang 1999, Theorem 1.6]). Let $f$ and $g$ be analytic in the interior of a simple closed Jordan curve $C$ and continuous on $C$, and let $|g(z)|<|f(z)|$ for all $z \in C$. Then $f$ and $f+g$ have the same number of zeros in the interior of the curve, counting multiplicities.

Definition 2.2. The following functions are defined for a given complex number $a$; positive integers $n$ and $k$ with $n \geq 3,1 \leq k \leq n-1$, and $\operatorname{gcd}(k, n)=1$; and $x \geq 0$ :

$$
\begin{aligned}
& \phi_{k}(x)=x^{n}+|a| x^{k}-1, \\
& \chi_{k}(x)=x^{n}-|a| x^{k}-1, \\
& \psi_{k}(x)=x^{n}-|a| x^{k}+1 .
\end{aligned}
$$

As the complex number $a$ remains the same throughout, we have omitted it from the notation. In the following definition, we define the separability threshold $\sigma(n, k)$, which, as will be shown in Lemma 2.4, is used to determine the number of positive zeros of $\psi_{k}$, which in turn determines if we can separate the zeros of $q$ into two groups of $k$ and $n-k$ zeros, whence $\sigma(k, n)$ 's name.

Definition 2.3. The separability threshold $\sigma(n, k)$ is defined for positive integers $k$ and $n$ (with $n \geq 3,1 \leq k \leq n-1$, and $\operatorname{gcd}(k, n)=1$ ) as

$$
\sigma(n, k)=\frac{n}{n-k}\left(\frac{n-k}{k}\right)^{k / n} \text {. }
$$

Since

$$
\sigma(n, n-k)=\frac{n}{k}\left(\frac{k}{n-k}\right)^{(n-k) / n}=\frac{n}{k}\left(\frac{k}{n-k}\right)\left(\frac{k}{n-k}\right)^{-k / n}=\sigma(n, k),
$$

$\sigma(n, k)$ is a symmetric function of $k$ for fixed $n$. Furthermore, $1<\sigma(n, k)<2$ and, for given $n$, achieves its maximum value at $k=\lceil n / 2\rceil-1,\lfloor n / 2\rfloor+1$, and its minimum value at $k=1, n-1$. We also have $\lim _{n \rightarrow+\infty} \sigma(n, 1)=1$.

Lemma 2.4. The following properties hold for the functions $\phi_{k}, \chi_{k}$, and $\psi_{k}$ from Definition 2.2.

(a) $\phi_{k}$ has a unique positive root $t$, and it lies on the interval $(0,1) ; \chi_{k}$ has a unique positive root $s$, and it lies on the interval $(1,+\infty)$; and the positive roots of $\phi_{k}$ and $\chi_{n-k}$ are each others' reciprocals.

(b) The function $\psi_{k}$ satisfies $\psi_{k}(0)>0$ and $\psi_{k}(|a|)>0$, it achieves its unique minimum on $(0,+\infty)$ at $\mu^{*}=(k|a| / n)^{1 /(n-k)}$, and $\psi_{k}\left(\mu^{*}\right)<0$ if and only if $|a|>\sigma(n, k)$. 

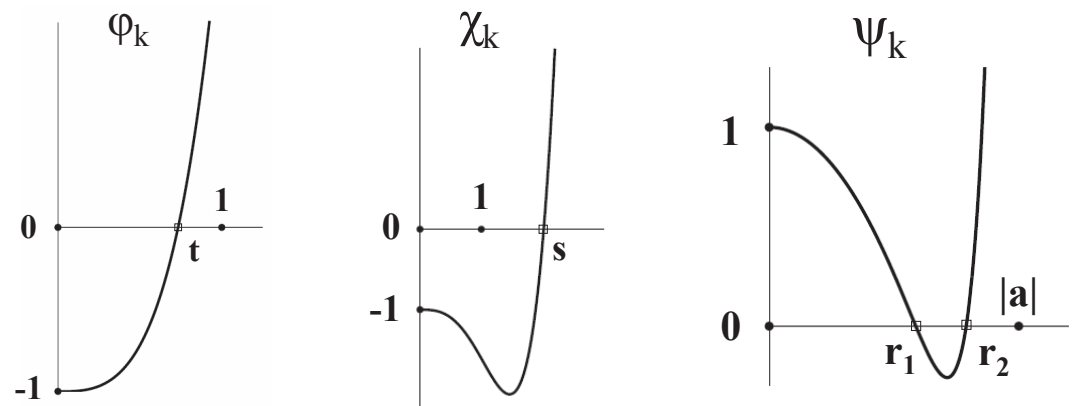

Figure 1. The functions $\phi_{k}, \chi_{k}$, and $\psi_{k}$.

(c) When $|a|>\sigma(n, k)$, then $\psi_{k}$ and $\psi_{n-k}$ both have two distinct positive roots. The roots of $\psi_{n-k}$ are the reciprocals of the roots of $\psi_{k}$. When $|a|<\sigma(n, k)$, then $\psi_{k}$ and $\psi_{n-k}$ have no positive roots.

The proof is an elementary exercise and will be omitted. The functions $\phi_{k}, \chi_{k}$, and $\psi_{k}$ are illustrated in Figure 1.

Lemma 2.5. (a) When $\sigma(n, k)<|a| \leq 2$, the two positive zeros $r_{1}$ and $r_{2}$ of $\psi_{k}$, with $r_{1}<r_{2}$, satisfy

$$
\begin{aligned}
& k>\frac{n}{2} \Rightarrow 1 \leq r_{1}<r_{2}<|a|, \\
& k<\frac{n}{2} \Longrightarrow 0<r_{1}<r_{2} \leq 1<|a| .
\end{aligned}
$$

(b) When $|a|>2$, the two positive zeros $r_{1}$ and $r_{2}$ of $\psi_{k}$ satisfy

$$
0<r_{1}<1<r_{2}<|a| \text {. }
$$

Proof. To understand where $r_{1}$ and $r_{2}$ are located when $\sigma(n, k)<|a| \leq 2$, we compute $\psi_{k}(1)=2-|a| \geq 0$, which means that we either have $1 \leq r_{1}<r_{2}<|a|$ or $0<r_{1}<r_{2} \leq 1<|a|$, depending on whether $\psi_{k}^{\prime}(1)<0$ or $\psi_{k}^{\prime}(1)>0$, respectively. Note that $\psi_{k}^{\prime}(1)=0$ is impossible because it would imply that the minimum is at 1 and therefore $\psi_{k}(1)<0$, contradicting our previous conclusion that $\psi_{k}(1) \geq 0$. Since $\psi_{k}^{\prime}(1)=n-k|a|$, we have

$$
\psi_{k}^{\prime}(1)>0 \Longleftrightarrow|a|<\frac{n}{k} \quad \text { and } \quad \psi_{k}^{\prime}(1)<0 \Longleftrightarrow|a|>\frac{n}{k} .
$$

However, given the upper limit on $|a|$ here, $|a|>n / k$ is impossible when $k<n / 2$. On the other hand, given the lower limit on $|a|,|a|<n / k$ cannot occur when $k>n / 2$ because then $k>n-k$, which would mean that $\sigma(n, k)=\sigma(n, n-k)>n / k$, and therefore $|a|>n / k$, a contradiction. We have therefore obtained that

$$
k>\frac{n}{2} \Longrightarrow \psi_{k}^{\prime}(1)<0 \text { and } k<\frac{n}{2} \Longrightarrow \psi_{k}^{\prime}(1)>0 \text {, }
$$


which proves the statement in part (a).

When $|a|>2$, we have $\psi_{k}(1)=2-|a|<0$, so that there will be one root strictly to either side of 1 .

Lemma 2.6. The positive roots $t$ and $s$ of the functions $\phi_{k}$ and $\chi_{k}$, respectively, and the positive roots $r_{1}$ and $r_{2}$ of $\psi_{k}$ (when they exist), with $r_{1}<r_{2}$, satisfy:

$$
(1+|a|)^{-1 / k}<t<r_{1}<\left(\frac{k}{n}|a|\right)^{1 /(n-k)}<r_{2}<s<(1+|a|)^{1 /(n-k)} .
$$

When $|a|>2$, then

$$
r_{1}<(|a|-1)^{-1 / k}<1<(|a|-1)^{1 /(n-k)}<r_{2} .
$$

Proof. For any $x \in(0,1), x^{n}<x^{k}$ so that $\phi_{k}(x)<(1+|a|) x^{k}-1$. Since $\phi_{k}$ is increasing, the root of the function on the right-hand side of this inequality, namely $(1+|a|)^{-1 / k}$, will be a strict lower bound on the positive root $t$ of $\phi_{k}$. Furthermore, by part (a) of Lemma 2.4, the positive roots of $\phi_{k}$ and $\chi_{n-k}$ are each others' reciprocals so that an upper bound on the positive root of $\chi_{k}$ is provided by the reciprocal of a lower bound on the positive root of $\phi_{n-k}$. Such a lower bound is, by what we just proved for $\phi_{k}$, given by $(1+|a|)^{-1 /(n-k)}$. Its reciprocal yields the strict upper bound on $s$.

Now assume that the roots $r_{1}$ and $r_{2}$ of $\psi_{k}$, with $r_{1}<r_{2}$, exist, and let $r=r_{1}$ or $r=r_{2}$. Then $r^{n}-|a| r^{k}+1=0$, or $|a| r^{k}=r^{n}+1$, implying that $\phi_{k}(r)=2 r^{n}>0$ and $\chi_{k}(r)=-2<0$ so that $t<r<s$. Consequently, $t<r_{1}<r_{2}<s$. Finally, the roots $r_{1}$ and $r_{2}$ lie on the left and right sides, respectively, of the minimum of $\psi_{k}$, which by part (b) of Lemma 2.4 is given by $(k|a| / n)^{1 /(n-k)}$.

When $|a|>2$, then $\psi_{k}(1)<0$, which implies that $r_{1}<1<r_{2}$. For $x \in(0,1)$, $\psi_{k}(x)<(1-|a|) x^{k}+1$, and $\psi_{k}$ is decreasing, so $r_{1}<(|a|-1)^{-1 / k}<1$. Since, by part (c) of Lemma 2.4 , the smallest root of $\psi_{n-k}$ is $1 / r_{2}$, we can find an upper bound on it as we just did for $r_{1}$. That upper bound is given by $(|a|-1)^{-1 /(n-k)}$. Its reciprocal then yields a lower bound on $r_{2}$.

All the bounds we derived are sufficient for our purposes, but they can be improved, if so desired. The numerical issues involved in this improvement are further elaborated in the Appendix.

In what follows, we will encounter sets of the form $L_{m}(c ; \rho)$, which we define as

$$
L_{m}(c ; \rho)=\left\{z \in \mathbb{C}:\left|z^{m}-c\right|<\rho\right\} .
$$

The boundary of $L_{m}(c ; \rho)$ is a lemniscate. When $|c|<\rho$, this lemniscate is a simple closed curve, and when $|c|>\rho$, it is a union of $m$ disconnected simple closed curves, which we will call loops. The interiors of the loops will be called leaves, with each leaf containing a different set of $m$-th roots of the complex numbers in 

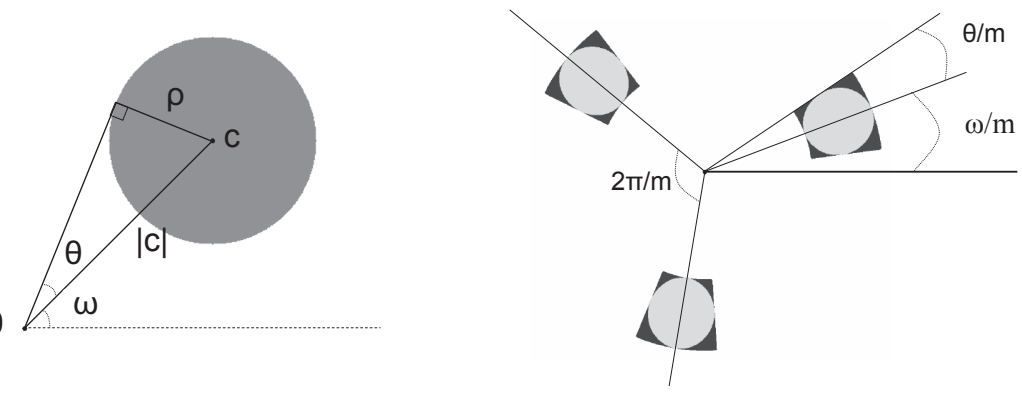

Figure 2. Left: $O(c ; \rho)$ (dark gray). Right: $L_{m}(c ; \rho)$ (light gray). In both cases, $m=3$.

$O(c ; \rho)$. It is, in general, cumbersome to carry out set operations on sets bounded by such lemniscates, but it is not difficult to enclose them by simpler sets. The following lemma shows how $L_{m}(c ; \rho)$ can be enclosed by a disk centered at the origin, or, when $|c|>\rho$, how its $m$ loops can be circumscribed by annular sectors.

Lemma 2.7. Let $m$ be a positive integer, $c \in \mathbb{C}$, and $\rho>0$. Then $O\left(0 ;(|c|+\rho)^{1 / m}\right)$ is the smallest disk centered at the origin that encloses $L_{m}(c ; \rho)$. Furthermore, when $|c|>\rho>0, \omega=\arg (c)$, and $\theta=\arcsin (\rho /|c|)$, then $L_{m}(c ; \rho)$ is circumscribed by the following union of disjoint annular sectors:

$$
\begin{aligned}
\left\{z \in \mathbb{C}: 0<(|c|-\rho)^{1 / m}<|z|<(|c|+\rho)^{1 / m},\right. & \\
& \left.\frac{\omega-\theta}{m}+j \cdot \frac{2 \pi}{m}<\arg (z)<\frac{\omega+\theta}{m}+j \cdot \frac{2 \pi}{m} \quad(j=0,1, \ldots, m-1)\right\} .
\end{aligned}
$$

Proof. Since $\left|z^{m}-c\right|<\rho$ implies $|z|^{m}<|c|+\rho, z \in L_{m}(c ; \rho)$ implies that $z \in O\left(0 ;(|c|+\rho)^{1 / m}\right)$ and it is the smallest such disk. When $|c|>\rho, z \in L_{m}(c ; \rho)$ implies $0<(|c|-\rho)^{1 / m}<|z|<(|c|+\rho)^{1 / m}$. With $\omega$ and $\theta$ as defined above, the argument of any $z \in L_{m}(c ; \rho)$ satisfies $\omega-\theta<\arg \left(z^{m}\right)<\omega+\theta$, as illustrated on the left in Figure 2. Taking the $m$-th root then completes the description of the enclosing set. The circumscribing annular sectors defined by this set, which are illustrated for $m=3$ on the right in Figure 2, are disjoint because $2 \theta / m<2 \pi / m$, as $\theta<\pi / 2$.

\section{Zero inclusion region valid for any value of $|a|$}

The following theorem derives upper and lower bounds on the moduli of $q$ 's zeros. We state it for the sake of completeness since, as a straightforward consequence of Rouché's theorem, it cannot be considered a new result.

Theorem 3.1. Let $q(z)=z^{n}-a z^{k}-1$, with integers $n \geq 3$ and $1 \leq k \leq n-1$ with $\operatorname{gcd}(k, n)=1$, and let $a \in \mathbb{C}$. Furthermore, let $s$ and $t$, with $0<t<1<s$, be 
the positive roots of $\chi_{k}(x)=x^{n}-|a| x^{k}-1=0$ and $\phi_{k}(x)=x^{n}+|a| x^{k}-1=0$, respectively. Then the zeros of $q$ are contained in the set

$$
\bar{O}(0 ; s) \cap O^{c}(0 ; t),
$$

that is, any such zero $z^{*}$ satisfies $t \leq\left|z^{*}\right| \leq s$.

Proof. If $\left|a z^{k}+1\right|<\left|z^{n}\right|$ holds on some circle $|z|=\rho$, then by Rouché's theorem, all of $q$ 's zeros are contained in the disk $O(0 ; \rho)$. Since the inequality holds if $|a||z|^{k}+1<|z|^{n}$ or $|z|^{n}-|a||z|^{k}-1<0$, any $\rho$ for which $\chi_{k}(\rho)<0$ or $\rho<s$ can be chosen. The zeros must then lie in the closed disk $\bar{O}(0 ; s)$.

For the lower bound, we observe that if $\left|z^{n}-a z^{k}\right|<1$ holds on some disk $|z|=\rho$, then, using Rouché's theorem once again, we conclude that none of $q$ 's zeros are contained in the disk $O(0 ; \rho)$. The inequality is satisfied if $|z|^{n}+|a||z|^{k}<1$ or $|z|^{n}+|a||z|^{k}-1<0$ so that an appropriate value for $\rho$ is any value for which $\phi_{k}(\rho)<0$ or $\rho<t$. All zeros must then lie in $O^{c}(0 ; t)$.

We remark that the same upper bound is also implied by a result of Cauchy [1829] (see also [Marden 1966, Theorem (27,1), p. 122]) and by Theorem I in [Kennedy 1940].

\section{Disjoint zero inclusion regions when $|a|>\sigma(n, k)$}

So far, we have obtained upper and lower bounds on the moduli of all zeros (Theorem 3.1) regardless of the value of $|a|$. In the following theorem, we use Rouché's theorem and Lemma 2.7 to derive $n$ disjoint annular sectors, each enclosing just one zero, when $|a|>\sigma(n, k)$.

Theorem 4.1. Let the zeros $\left\{z_{i}\right\}_{i=1}^{n}$ of $q(z)=z^{n}-a z^{k}-1$ (with integers $n \geq 3$ and $1 \leq k \leq n-1, \operatorname{gcd}(k, n)=1$, and $|a|>\sigma(n, k))$ be labeled in a way so that $\left|z_{1}\right| \leq \cdots \leq\left|z_{n}\right|$. Let $\delta_{1}$ and $\delta_{2}$ be such that $r_{1}<\delta_{1} \leq \delta_{2}<r_{2}$, where $r_{1}$ and $r_{2}$ are the positive roots of $x^{n}-|a| x^{k}+1=0$. Furthermore, let $\omega=\arg (a)$, $\theta_{1}=\arcsin \left(\delta_{1}^{n-k} /|a|\right)$, and $\theta_{2}=\arcsin \left(\delta_{2}^{-k} /|a|\right)$.

Then the first $k$ zeros $\left\{z_{i}\right\}_{i=1}^{k}$ are contained in the set

$$
\begin{aligned}
\Omega_{1}(q)= & \left\{z \in \mathbb{C}:\left(|a|+\delta_{1}^{n-k}\right)^{-1 / k}<|z|<\left(|a|-\delta_{1}^{n-k}\right)^{-1 / k},\right. \\
& \left.\frac{2 j \pi-\omega-\pi}{k}-\frac{\theta_{1}}{k}<\arg (z)<\frac{2 j \pi-\omega-\pi}{k}+\frac{\theta_{1}}{k} \quad(j=0,1, \ldots, k-1)\right\},
\end{aligned}
$$

while the remaining $n-k$ zeros $\left\{z_{i}\right\}_{i=k+1}^{n}$ are contained in the set

$$
\begin{aligned}
& \Omega_{2}(q)=\left\{z \in \mathbb{C}:\left(|a|-\delta_{2}^{-k}\right)^{1 /(n-k)}<|z|<\left(|a|+\delta_{2}^{-k}\right)^{1 /(n-k)},\right. \\
& \left.\frac{2 j \pi+\omega}{n-k}-\frac{\theta_{2}}{n-k}<\arg (z)<\frac{2 j \pi+\omega}{n-k}+\frac{\theta_{2}}{n-k} \quad(j=0,1, \ldots, n-k-1)\right\} .
\end{aligned}
$$


We also have

$$
\left(|a|-\delta_{1}^{n-k}\right)^{-1 / k}<\delta_{1} \leq \delta_{2}<\left(|a|-\delta_{2}^{-k}\right)^{1 /(n-k)} .
$$

In addition, all the annular sectors in $\Omega_{1}(q)$ and $\Omega_{2}(q)$ are disjoint and each contains exactly one zero of $q$.

Proof. If $\left|z^{n}-1\right|<\left|a z^{k}\right|$ holds on some circle $|z|=\rho$, then by Rouché's theorem, $k$ of $q$ 's zeros are contained in the disk $O(0 ; \rho)$. Because the inequality holds if $|z|^{n}+1<|a||z|^{k}$ or $|z|^{n}-|a||z|^{k}+1<0$, any $\rho$ for which $\psi_{k}(\rho)<0$ or $r_{1}<\rho<r_{2}$ can be chosen. Since $r_{1}<\delta_{1} \leq \delta_{2}<r_{2}$, we conclude that $k$ zeros lie in $O\left(0 ; \delta_{1}\right)$, while the remaining $n-k$ zeros lie in the complement of $\bar{O}\left(0 ; \delta_{2}\right)$. This also follows from Pellet's theorem [Marden 1966, Theorem $(28,1)]$.

Since $z^{n}-a z^{k}-1=0$ implies that $|z|^{k}\left|z^{n-k}-a\right|=1$ and, as a result, that $\left|z^{n-k}-a\right|=1 /|z|^{k}$, the $(n-k)$-th powers of the $n-k$ largest zeros $\left\{z_{i}\right\}_{i=k+1}^{n}$, all of which satisfy $\left|z_{i}\right|>\delta_{2}$, must lie in $O\left(a, \delta_{2}^{-k}\right)$. Moreover, since $\delta_{2}^{n}-|a| \delta_{2}^{k}+1<0$, we have $\delta_{2}^{n-k}+\delta_{2}^{-k}<|a|$ so that $|a|>\delta_{2}^{-k}$, which in turn implies that $O\left(a ; \delta_{2}^{-k}\right)$ is bounded away from the origin. The zeros themselves must lie in $L_{n-k}\left(a, \delta_{2}^{-k}\right)$, which, from Lemma 2.7 with $m=n-k, c=a$, and $\rho=\delta_{2}^{-k}$, consists of $n-k$ disjoint leaves that are contained in $\Omega_{2}(q)$, a set consisting of disjoint annular sectors. That $\left(|a|-\delta_{2}^{-k}\right)^{1 /(n-k)}>\delta_{2}$ follows from $\delta_{2}^{n}-|a| \delta_{2}^{k}+1<0$. Consequently, any $z \in \Omega_{2}(p)$ satisfies $|z|>\delta_{2}$.

We now prove that each leaf of $L_{n-k}\left(a, \delta_{2}^{-k}\right)$ contains exactly one zero. For this, we appeal once more to Rouché's theorem. Recall that $L_{n-k}\left(a ; \delta_{2}^{-k}\right) \subseteq \Omega_{2}(p)$ so that $|z|>\delta_{2}$ for any $z \in L_{n-k}\left(a ; \delta_{2}^{-k}\right)$, which is composed of $n-k$ disjoint closed leaves bounded away from the origin, each containing a different $(n-k)$-th root of $a$. For any $z$ on the (Jordan) boundary curve of one of these leaves, we have $\left|z^{n-k}-a\right|=\delta_{2}^{-k}$, so that $\left|z^{n}-a z^{k}\right|=|z|^{k} \delta_{2}^{-k}>1$. By Rouché's theorem, this means that the polynomial $z^{n}-a z^{k}-1$ has as many zeros strictly inside this leaf as $z^{n}-a z^{k}$ and that number is exactly one since it contains just one $(n-k)$-th root of $a$ and it does not contain zero. There are $n-k$ disjoint annular sectors each containing such a leaf and therefore each contains at least one zero of $q$. Since there are $n-k$ such zeros, each annular sector must contain exactly one zero.

For the first $k$ zeros $\left\{z_{i}\right\}_{i=1}^{k}$, we consider the fact that $z^{n}-a z^{k}-1=0$ implies that $\left|-a z^{k}-1\right|=\left|z^{n}\right|$ and therefore that $\left|(-a)-z^{-k}\right|=|z|^{n-k}$. Because now $\left|z_{i}\right|<\delta_{1}$, this means that the $k$-th powers of the reciprocals of the $k$ smallest zeros must lie in $O\left(-a, \delta_{1}^{n-k}\right)$. Also, $\delta_{1}^{n}-|a| \delta_{1}^{k}+1<0$, so $\delta_{1}^{n-k}+\delta_{1}^{-k}<|a|$. Therefore $|a|>\delta_{1}^{n-k}$, implying that $O\left(-a, \delta_{1}^{n-k}\right)$ is bounded away from the origin. Analogously to what we did for the $n-k$ largest zeros, we now apply Lemma 2.7 with $m=k, c=-a$, and $\rho=\delta_{1}^{n-k}$, from which we obtain that the reciprocals of the zeros themselves must lie in $L_{k}\left(-a, \delta_{1}^{n-k}\right)$, which consists of $k$ disjoint leaves that are contained in 
the set of disjoint annular sectors $\Omega_{1}(q)$. The latter follows from

$$
\frac{1}{|z|}<\left(|a|+\delta_{1}^{n-k}\right)^{1 / k} \Longrightarrow|z|>\left(|a|+\delta_{1}^{n-k}\right)^{-1 / k},
$$

and also

$$
\frac{1}{|z|}>\left(|a|-\delta_{1}^{n-k}\right)^{1 / k} \Rightarrow|z|<\left(|a|-\delta_{1}^{n-k}\right)^{-1 / k}<\delta_{1},
$$

with the last inequality following from $\delta_{1}^{n}-|a| \delta_{1}^{k}+1<0$. The bounds on the arguments in the definition of $\Omega_{1}(q)$ are then determined from $\arg (z)=-\arg (1 / z)$ and $\arg (-a)=\arg (a)+\pi$.

That each annular sector in $\Omega_{1}(q)$ contains exactly one zero follows from similar arguments as for the largest $n-k$ zeros of $q$. For any $z$ on the boundary of any one of the leaves, we have

$$
\left|-a-z^{-k}\right|=\delta_{1}^{n-k} \Longrightarrow\left|a z^{k}+1\right|=|z|^{k} \delta_{1}^{n-k}>|z|^{n} .
$$

Rouché's theorem then implies that $z^{n}-a z^{k}-1$ has as many zeros strictly inside this leaf as $a z^{k}+1$, which is exactly one and that each annular sector must contain a single zero, just as in the case for the $n-k$ largest zeros.

Remarks 4.2. (1) We note that easily computed explicit values for the bounds involved in the definitions of the annular sectors are given by Lemma 2.6.

(2) The smallest $\Omega_{1}(q)$ and $\Omega_{2}(q)$ are obtained by replacing $\delta_{1}$ and $\delta_{2}$ by $r_{1}$ and $r_{2}$, respectively, and by replacing the strict inequalities by inclusive inequalities in their definitions.

(3) The lower bound on $|z|$ in $\Omega_{1}(q)$ and the upper bound on $|z|$ in $\Omega_{2}$ can be replaced by $t$ and $s$, respectively, as defined in Theorem 3.1, to yield better bounds. Let us consider the upper bound. The inequality $\left(|a|+r_{2}^{-k}\right)^{1 /(n-k)}>s$ is equivalent to $|a|+r_{2}^{-k}>s^{n-k}$, which in turn is equivalent to $s^{n}-|a| s^{k}-\left(s / r_{2}\right)^{k}<0$. That this inequality holds follows from the fact that $r_{2}<s$ (by Lemma 2.6) and that $s^{n}-|a| s^{k}-1=0$. The lower bound in $\Omega_{1}(q)$ is analogously shown to be improved by $t$.

(4) We note that the angles of the annular sectors in Theorem 4.1 depend on $|a|$ and decrease with increasing $|a|$. This is qualitatively different from previous results in, for example, [Biernacki 1928; Herglotz 1922; Kempner 1922; Nekrassoff 1887, Satz II], where these angles are rigid and insensitive to $a$, making them significantly larger (and therefore worse) than the sectors in Theorem 4.1 as $|a|$ increases. The radii of the annular sectors we obtained are also better than the ones in [Nekrassoff 1887], as will be illustrated in the examples later on.

(5) Asymptotic upper bounds on the angles $\theta_{1}$ and $\theta_{2}$ for $|a| \rightarrow+\infty$ can be determined with the help of Lemma 2.6, which shows that in that case one can choose 
$\delta_{1}=(|a|-1)^{-1 / k}$ and $\delta_{2}=(|a|-1)^{1 / n-k}$, implying that

$$
\begin{aligned}
\lim _{|a| \rightarrow+\infty} \theta_{1}(a) & =\lim _{|a| \rightarrow+\infty} \arcsin \left(\delta_{1}^{n-k} /|a|\right) \\
& \leq \lim _{|a| \rightarrow+\infty} \arcsin \left((|a|-1)^{-(n-k) / k} /|a|\right) \\
& \leq \lim _{|a| \rightarrow+\infty} \arcsin \left(|a|^{-(n-k) / k} /|a|\right) \\
& \leq|a|^{-n / k}+O\left(|a|^{-3 n / k}\right),
\end{aligned}
$$

and, analogously, that

$$
\lim _{|a| \rightarrow+\infty} \theta_{2}(a) \leq|a|^{-n /(n-k)}+\mathscr{O}\left(|a|^{-3 n /(n-k)}\right) .
$$

This means that both $\theta_{1}$ and $\theta_{2}$ converge to zero as $|a| \rightarrow+\infty$ at a rate determined by $n$ and $k$ and that the slower that convergence is for one angle, the faster it will be for the other. A tedious, but similar, computation for the differences $\Delta_{1}(a)$ and $\Delta_{2}(a)$ between the radii of the annular sectors in $\Omega_{1}(q)$ and in $\Omega_{2}(q)$, respectively, shows that $\Delta_{1}(a) \sim \mathcal{O}\left(|a|^{-(n+1) / k}\right)$ and $\Delta_{2}(a) \sim \mathcal{O}\left(|a|^{-(n-1) /(n-k)}\right)$ as $|a| \rightarrow+\infty$.

This corollary is an immediate consequence of Theorem 4.1:

Corollary 4.3. Let the zeros $\left\{z_{i}\right\}_{i=1}^{n}$ of $q(z)=z^{n}-a z^{k}-1$ (with integers $n \geq 3$ and $1 \leq k \leq n-1$ and $\operatorname{gcd}(k, n)=1)$ be labeled so that $\left|z_{1}\right| \leq \cdots \leq\left|z_{n}\right|$.

(a) When $\sigma(n, k)<|a| \leq 2$ and $k>n / 2$, then $\left|z_{i}\right|>1$ for $i=k+1, \ldots, n$.

(b) When $\sigma(n, k)<|a| \leq 2$ and $k<n / 2$, then $\left|z_{i}\right|<1$ for $i=1, \ldots, k$.

(c) When $|a|>2$, then $\left|z_{i}\right|<1$ for $i=1, \ldots, k$ and $\left|z_{i}\right|>1$ for $i=k+1, \ldots, n$.

Proof. When $\sigma(n, k)<|a| \leq 2$ and $k>n / 2$, Lemma 2.5 says $1 \leq r_{1}<r_{2}<|a|$. From Theorem 4.1 with $r_{1}<\delta_{1} \leq \delta_{2}<r_{2}$, one then obtains that the $n-k$ zeros $\left\{z_{i}\right\}_{i=k+1}^{n}$ are contained in $\Omega_{2}(p)$ and therefore satisfy

$$
\left|z_{i}\right|>\left(|a|-\delta_{2}^{-k}\right)^{1 /(n-k)}>\delta_{2}>r_{1} \geq 1 .
$$

When $\sigma(n, k)<|a| \leq 2$ and $k<n / 2$, Lemma 2.5 yields that $0<r_{1}<r_{2} \leq 1<|a|$. From Theorem 4.1 with $r_{1}<\delta_{1} \leq \delta_{2}<r_{2}$, one then obtains that the $k$ zeros $\left\{z_{i}\right\}_{i=1}^{k}$ are contained in $\Omega_{1}(p)$ and therefore satisfy

$$
\left|z_{i}\right|<\left(|a|-\delta_{1}^{n-k}\right)^{-1 / k}<\delta_{1}<r_{2} \leq 1 .
$$

When $|a|>2$, Lemma 2.5 yields that $0<r_{1}<1<r_{2}<|a|$. From Theorem 4.1 with $\delta_{1}=\delta_{2}=1$, one then obtains that the $k$ zeros $\left\{z_{i}\right\}_{i=1}^{k}$ are contained in $\Omega_{1}(p)$ and therefore satisfy

$$
\left|z_{i}\right|<(|a|-1)^{-1 / k}<1
$$


and that the $n-k$ zeros $\left\{z_{i}\right\}_{i=k+1}^{n}$ are contained in $\Omega_{2}(p)$ and therefore satisfy

$$
\left|z_{i}\right|>(|a|-1)^{1 /(n-k)}>1 \text {. }
$$

Examples 4.4. To illustrate the zero inclusion regions in Theorem 4.1, we consider the trinomial $q_{1}(z)=z^{10}-(1.6+i) z^{7}-1$, for which $a=1.6+i,|a|=1.8868$, and $\sigma(10,7)=1.8420$ so that $\sigma(10,7)<|a|<2$. From Theorem 4.1, we can therefore expect an inclusion region composed of disjoint annular sectors. The moduli of seven of the zeros of $q_{1}$ lie in the interval [0.8746, 0.9771], whereas three have their moduli in the interval [1.2140, 1.2739]. Figure 3 shows, from left to right and using the same terminology as before, the shaded inclusion regions: (4-1) when using the bounds from Lemma 2.6; (4-2) when using bounds, obtained after only two Newton steps for the computation of $s$ and $t$ and only one step of the method outlined in the Appendix, with the initial points chosen as the bounds from Lemma 2.6; and (4-3) when using the exact values of $s, t, r_{1}$, and $r_{2}$. The corresponding estimated ranges for the moduli of the first seven and last three zeros are, respectively,

$$
0.8595 \text { to } 1.0847 \text { and } 1.1091 \text { to } 1.4239 \text {, }
$$

$$
0.8745 \text { to } 1.0639 \text { and } 1.1236 \text { to } 1.2803 \text {, }
$$

$$
0.8746 \text { to } 1.0389 \text { and } 1.1438 \text { to } 1.2744 \text {. }
$$

The small black dots are the zeros. The shaded regions become smaller from left to right.

The corresponding (exact) optimal bounds obtained in [Nekrassoff 1887], involving the solution of nonlinear equations similar to the ones in Definition 2.2, are

$$
0.8735 \text { to } 1.0586 \text { and } 1.0972 \text { to } 1.2901 \text {. }
$$

The sectors obtained in [Nekrassoff 1887] strictly contain the ones obtained from Theorem 4.1. In [Biernacki 1928], the upper bound on the seven smallest zeros is the same as the one obtained from the bound in Lemma 2.6. The results in [Landau 1906; 1907] are not applicable here, as they are only valid for $k=1$.
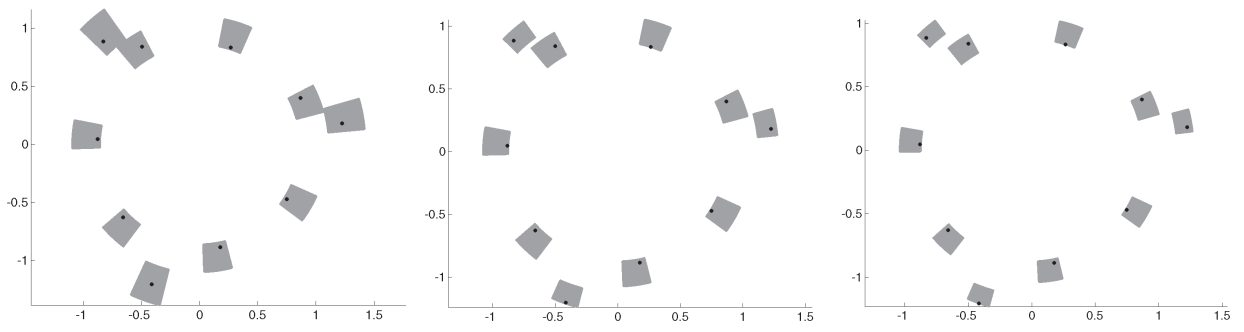

Figure 3. Zero inclusion regions for $q_{1}$. 

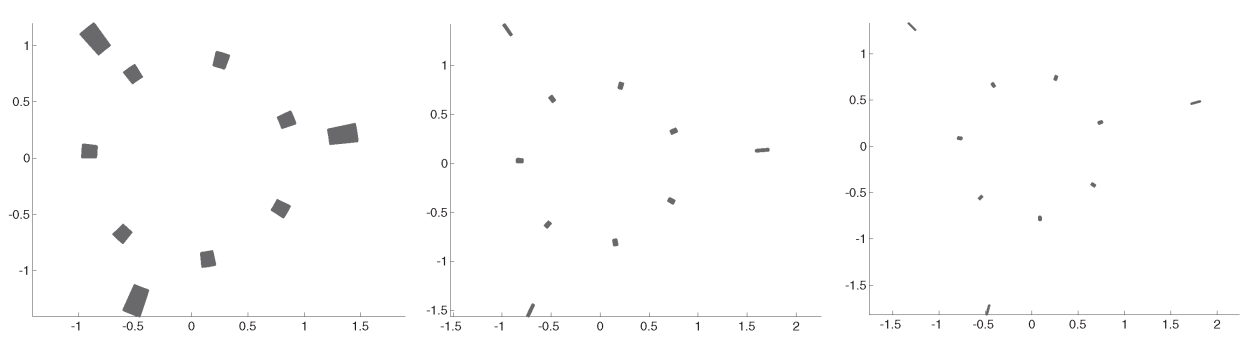

Figure 4. Zero inclusion regions for $n=10, k=7$ when $a=2+i$, $a=4+i$, and $a=4+4 i$.

As $|a|$ becomes larger, the angles subtended by the annular sectors containing the zeros shrink, unlike the sectors in previous results, for example, [Biernacki 1928; Herglotz 1922; Kempner 1922; Nekrassoff 1887]. Figure 4 shows, shaded in dark gray and from left to right, the significant decrease in the size of our inclusion regions for a trinomial with $n=10$ and $k=7$, when $a=2+i, a=4+i$, and $a=4+4 i$, respectively, where we just used the simple bounds from Lemma 2.6. The zeros are not indicated to avoid obscuring the small inclusion regions.

\section{Disjoint zero inclusion regions when $|a|<\sigma(n, k)$}

We now turn our attention to the case $|a|<\sigma(n, k)$, for which the following theorem establishes contiguous annular sectors, each containing one zero of $z^{n}-a z^{k}-1$, and whose subtended angles are the same as the ones obtained in [Nekrassoff 1887, Satz I]. It is stated here for completeness, as we will need it later on. Our proof is different from the one in [Nekrassoff 1887] since it relies on a straightforward application of Rouché's theorem, which was not used in [Nekrassoff 1887] and which, as will be illustrated in the examples that are to follow, also improves the radii of the annular sectors.

Theorem 5.1. Let $q(z)=z^{n}-a z^{k}-1$, with integers $n \geq 3$ and $1 \leq k \leq n-1$, $\operatorname{gcd}(k, n)=1$, and $|a|<\sigma(n, k)$. Let $s$ and $t$ be the positive roots of the real equations $x^{n}-|a| x^{k}-1=0$ and $x^{n}+|a| x^{k}-1=0$, respectively, and consider the $n$ contiguous annular sectors, each bounded by arcs of radius $\rho_{1}$ such that $\rho_{1}<t<1$ and $\rho_{2}$ such that $\rho_{2}>s>1$, with subtended angle $2 \pi / n$, and centered at the angle $2 \ell \pi / n$ for $\ell=0,1, \ldots, n-1$. Then each of these annular sectors contains exactly one zero of $q$ in its interior.

Proof. The boundary of each annular sector consists of two arcs and the two segments connecting them, and each contains a single $n$-th root of 1 . Now, for a given integer $\ell$, consider the annular sector centered at the angle $2 \ell \pi / n$ that contains the $n$-th root of unity $\exp (2 \ell \pi i / n)$. For $z$ on the inner arc, we have $z=\rho_{1} \exp (i \zeta)$, with $2 \ell \pi / n-\pi / n \leq \zeta \leq 2 \ell \pi / n+\pi / n$. Therefore, when $z$ moves along the inner 
arc from one endpoint to the other, $z^{n}$ describes a full circle of radius $\rho_{1}^{n}$ around the origin so that $\left|z^{n}-1\right| \geq 1-\rho_{1}^{n}$. Because of the definition of $\rho_{1}$, illustrated by Figure 1, we then have on this arc that

$$
\left|a z^{k}\right|=|a| \rho_{1}^{k}<1-\rho_{1}^{n} \leq\left|z^{n}-1\right| .
$$

Analogously, when $z$ lies on the outer arc, we obtain from the definition of $\rho_{2}$ and, once again illustrated by Figure 1, that

$$
\left|a z^{k}\right|=|a| \rho_{2}^{k}<\rho_{2}^{n}-1 \leq\left|z^{n}-1\right| .
$$

On the segments connecting the arcs, $z=x \exp (2 \ell \pi i / n \pm \pi i / n)$ with $\rho_{1} \leq x \leq \rho_{2}$ so that $z^{n}=x^{n} \exp (2 \ell \pi i \pm \pi i)=-x^{n}$, from which we obtain that $\left|z^{n}-1\right|=x^{n}+1$. Since $|a|<\sigma(n, k)$, we have from part (c) in Lemma 2.4 that $x^{n}-|a| x^{k}+1>0$ for $x \geq 0$, and therefore,

$$
\left|a z^{k}\right|=|a| x^{k}<x^{n}+1=\left|z^{n}-1\right| .
$$

Inequalities (5-1), (5-2), and (5-3) imply that $\left|a z^{k}\right|<\left|z^{n}-1\right|$ on the boundary of the annular sector containing the single $n$-th root of unity $\exp (2 \ell \pi i / n)$. By Rouché theorem, that means that this annular sector contains exactly one zero of $z^{n}-a z^{k}-1$ in its interior.

Theorem 5.1 establishes $n$ open contiguous annular sectors each containing a zero of the polynomial $q$, but the angles subtended by these annular sectors depend only on $n$ and are therefore fixed, regardless of the value of the coefficient $a$. However, as $|a| \rightarrow 0$, the zeros of $q$ converge to the $n$-th roots of unity. The annular sectors in Theorem 5.1 thus fail to capture a fundamental property of the zeros. In what follows we will remedy this situation by refining Theorem 5.1, for which we will need this simple lemma:

Lemma 5.2. Let $\alpha_{1}$ and $\alpha_{2}$ be the positive roots of $x^{n}-c x^{k_{1}}-1$ and $x^{n}-c x^{k_{2}}-1$, respectively, with $c>0$ and $1 \leq k_{1}, k_{2} \leq n-1$. Then $k_{1}<k_{2} \Longrightarrow \alpha_{1}<\alpha_{2}$.

Proof. Assume that $k_{1}<k_{2}$ and define $f_{j}(x)=x^{n}-c x^{k_{j}}-1(j=1,2)$ so that $f_{j}\left(\alpha_{j}\right)=0$ and $f_{j}(x)<0$ for $x<\alpha_{j}$. The functions $f_{j}$ are of the same form as $\phi_{k}$ so that part (a) in Lemma 2.4 implies that $\alpha_{1}, \alpha_{2}>1$ and therefore that $f_{2}\left(\alpha_{1}\right)<f_{1}\left(\alpha_{1}\right)=0$. Since $f_{2}\left(\alpha_{2}\right)=0$, this means that $f_{2}\left(\alpha_{1}\right)<f_{2}\left(\alpha_{2}\right)$. Because $f_{2}$ is strictly increasing on $(0,+\infty)$, we conclude that $\alpha_{1}<\alpha_{2}$.

Theorem 5.3. Let $q(z)=z^{n}-a z^{k}-1$, with integers $n \geq 3$ and $1 \leq k \leq n-1$ and $\operatorname{gcd}(k, n)=1$. Let $\rho_{1}$ and $\rho_{2}$ be such that $0<\rho_{1}<t<1<s<\rho_{2}$, where $s$ and $t$ are the positive roots of $x^{n}-|a| x^{k}-1=0$ and $x^{n}+|a| x^{k}-1=0$, respectively. Set $\tau=\min (k, n-k)$, and let $u$ be the positive zero of $x^{n}-|a| x^{\tau}-1=0$. 
(a) If $|a|<2^{-\tau / n}$, then there exists $\bar{u} \geq u$ such that $|a| \bar{u}^{\tau}<1$, in which case, with $\theta=\arcsin \left(|a| \bar{u}^{\tau}\right)$, the zeros of $q$ are contained in the set

$$
\begin{aligned}
\Omega(q)=\{z \in \mathbb{C}: & \rho_{1}<|z|<\rho_{2}, \\
& \left.\frac{2 j \pi}{n}-\frac{\theta}{n} \leq \arg (z) \leq \frac{2 j \pi}{n}+\frac{\theta}{n} \quad(j=0,1, \ldots, n-1)\right\},
\end{aligned}
$$

which is composed of $n$ disjoint annular sectors. Each annular sector contains exactly one zero of $q$.

(b) If $|a|(1+|a|)^{\tau /(n-\tau)}<1$, then we may use $\bar{u}=(1+|a|)^{1 /(n-\tau)} \geq u$, so $|a| \bar{u}^{\tau}<1$. This condition on $|a|$ is always satisfied when $|a| \leq 2(1+\sqrt{5})^{-1} \approx 0.618$.

Proof. We begin by observing that $|a|<2^{-\tau / n}$ implies that $|a|<1$ and therefore that $|a|<\sigma(n, k)$. The bounds on $|z|$ in the definition of $\Omega(q)$ follow from Theorem 5.1, but they also follow directly from Theorem 3.1, which states that $t \leq\left|z_{i}\right| \leq s$ for any zero $z_{i}(1 \leq i \leq n)$ of $q$.

The reciprocal polynomial of $q$ is $Q(z)=z^{n}+a z^{n-k}-1$; its zeros are $1 / z_{i}$ $(1 \leq i \leq n)$. Applying Theorem 3.1 to $Q$ yields $\left|1 / z_{i}\right| \leq \tilde{s}$, where $\tilde{s}$ is the positive root of $x^{n}-|a| x^{n-k}-1$. From $q\left(z_{i}\right)=0$ we obtain that $\left|z_{i}^{n}-1\right|=|a|\left|z_{i}\right|^{k} \leq|a| s^{k}$ and from $Q\left(1 / z_{i}\right)=0$ that $\left|\left(1 / z_{i}\right)^{n}-1\right|=|a|\left(1 /\left|z_{i}\right|\right)^{n-k} \leq|a| \tilde{s}^{n-k}$, which shows that the $n$-th powers of $z_{i}$ and $1 / z_{i}$ each lie in a disk centered at 1 . The zeros and their reciprocals themselves then lie in a lemniscate composed of disjoint leaves, each leaf containing a different $n$-th root of unity, if their corresponding disk is bounded away from the origin. In that case, bounds on the arguments of the zeros can easily be obtained, just like before. Since $\arg \left(1 / z_{i}\right)=-\arg \left(z_{i}\right)$, either disk can be used. To obtain the best possible bounds on the arguments, we therefore choose the disk with the smallest radius. From Lemma 5.2 this will be the disk with radius $|a| s^{k}$ when $k<n-k$ or the disk with radius $|a| \tilde{s}^{n-k}$ when $n-k<k$. In other words, the smallest radius is given by $|a| u^{\tau}$, where $\tau=\min (k, n-k)$ and $u$ is the positive root of $x^{n}-|a| x^{\tau}-1$.

Therefore, when $|a| \bar{u}^{\tau}<1$ for some $\bar{u}$ such that $\bar{u} \geq u$, we have from Lemma 2.7 with $m=n, c=1$, and $\rho=|a| \bar{u}^{\tau}$ that the aforementioned disjoint leaves are circumscribed by disjoint annular sectors, each subtending an angle of $2 \theta / n$ with $\theta=\arcsin \left(|a| \bar{u}^{\tau}\right)$. The annular sector containing $\exp (2 j \pi i / n)$, the $n$-th root of unity, for an integer $j(0 \leq j \leq n-1)$ is symmetric about a ray emanating from the origin and forming an angle of $2 j \pi / n$ with the real axis. These are precisely the disjoint annular sectors of $\Omega(q)$. Applying Theorem 5.1, we observe that each annular sector defined by that theorem contains exactly one of the annular sectors of $\Omega(q)$, and, because each annular sector in Theorem 5.1 contains exactly one zero of $q$, this zero must necessarily lie in the corresponding annular sector of $\Omega(q)$. 
To find a condition on $|a|$ for which $|a| u^{\tau}<1$, we note that $u^{n}-|a| u^{\tau}-1=0$ so that the condition is equivalent to $u^{n}-1<1$ and therefore to $u<2^{1 / n}$. This requires that $\left(2^{1 / n}\right)^{n}-|a|\left(2^{1 / n}\right)^{\tau}-1>0$, or, equivalently, $|a|<2^{-\tau / n}$. A continuity argument then ensures that for any such $a$ there exists $\bar{u} \geq u$ such that $|a| \bar{u}^{\tau}<1$.

For part (b), we use the upper bound on $s$ from Lemma 2.6 with $s=u$ and $k=\tau$ to obtain that $u \leq(1+|a|)^{1 /(n-\tau)}$. Setting $\bar{u}=(1+|a|)^{1 /(n-\tau)}$ then implies, given the assumption in the statement of the theorem, that $\bar{u} \geq u$ and $|a| \bar{u}^{\tau}<1$. Furthermore, we observe that $\tau<n / 2$ and therefore $\tau /(n-\tau)<1$ so that

$$
|a|(1+|a|)^{\tau /(n-\tau)}<|a|(1+|a|) .
$$

Requiring that $|a|(1+|a|) \leq 1$ then leads to $|a| \leq(-1+\sqrt{5}) / 2=2(1+\sqrt{5})^{-1}$.

Remarks 5.4. (1) Easily computable values for the bounds are, once again, obtained from Lemma 2.6.

(2) The value $2^{-\tau / n}$, like $\sigma(n, k)$, is symmetric in $k$ and satisfies $2^{-1 / 2}<2^{-\tau / n}<1$.

(3) Although for brevity's sake we will refrain from doing so, it can be shown that the radii of the annular sectors that we obtained both in Theorem 4.1 and in Theorem 5.3 are better than the corresponding ones in [Nekrassoff 1887].

(4) Theorem 5.3 establishes annular sectors that depend on $a$ and whose subtended angles converge to zero as $a \rightarrow 0$. This is in sharp contrast to the angles subtended by the annular sectors of Satz I in [Nekrassoff 1887], which are impervious to changes in $a$. Our result is valid when $|a| u^{\tau}<1$, which holds for-but is not limited to-any $a$ satisfying $|a| \leq 0.618$.

(5) An asymptotic upper bound on $\theta$ for $|a| \rightarrow 0$ can be determined by

$$
\begin{aligned}
\lim _{|a| \rightarrow 0} \theta(a) & =\lim _{|a| \rightarrow 0} \arcsin \left(|a| u^{\tau}\right) \\
& \leq \lim _{|a| \rightarrow 0} \arcsin \left(|a|(1+|a|)^{\tau /(n-\tau)}\right) \leq|a|+\mathcal{O}\left(|a|^{2}\right) .
\end{aligned}
$$

A simple similar computation for the difference $\Delta(a)$ between the radii of the annular sectors in $\Omega(q)$ shows that $\Delta(a) \sim \mathcal{O}(|a|)$ as $|a| \rightarrow 0$.

Examples 5.5. We now illustrate the zero inclusion regions from Theorem 5.3 by considering a trinomial with $n=10$ and $k=7$ as before, this time with small values for $|a|$. It is given by $q_{2}(z)=z^{10}-(0.4+0.6 i) z^{7}-1$, for which $a=0.4+0.6 i$, $|a|=0.7211, \sigma(10,7)=1.8420, \tau=3$, and $2^{-3 / 10}=0.8123$, so that $|a|<\sigma(n, k)$, and $|a|<2^{-\tau / n}$. From Theorem 5.3, we can therefore expect an inclusion region composed of ten disjoint annular sectors. The moduli of the zeros of $q_{2}$ lie in the interval $[0.9407,1.0818]$. Figure 5 shows, from left to right and using the same terminology as before, the shaded inclusion regions: (5-5) when using the bounds from Lemma 2.6; (5-6) when using bounds, obtained after only two Newton steps 


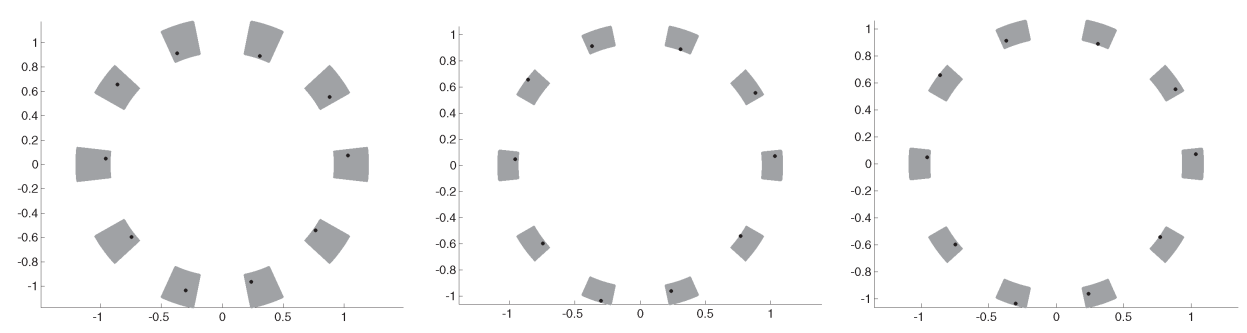

Figure 5. Zero inclusion regions for $q_{2}$.

for the computation of $s$ and $t$ and only one step of the method outlined in the Appendix, with the initial points chosen as the bounds from Lemma 2.6; and (5-7) when using the exact values of $s, t$, and $u$. The corresponding estimated ranges for the moduli of the zeros are, respectively,

$$
0.9254 \text { to } 1.1984 \text {, }
$$$$
0.9393 \text { to } 1.0875 \text {, }
$$

$$
0.9393 \text { to } 1.0862 \text {. }
$$

The small black dots are the zeros. The shaded regions become smaller from left to right.

The corresponding (exact) optimal bounds obtained in [Nekrassoff 1887], involving the solution of nonlinear equations similar to the ones in Definition 2.2, are

$$
0.8765 \text { to } 1.2668 \text {. }
$$

The sectors obtained in [Nekrassoff 1887] strictly contain the ones obtained from Theorem 5.3. The other references mentioned in the examples of the previous section are not applicable here.

This time, as $|a|$ becomes smaller, the angles subtended by the annular sectors containing the zeros shrink, unlike the sectors in [Nekrassoff 1887]. Figure 6 shows, shaded in dark gray and from left to right, the significant decrease in the size
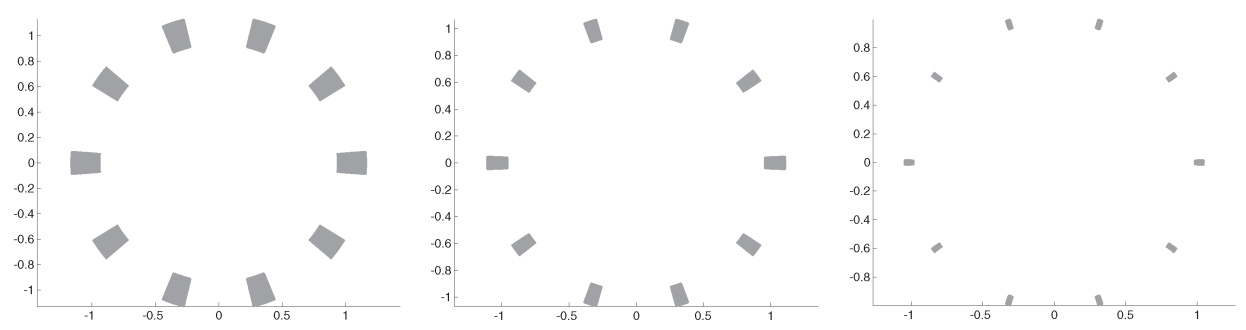

Figure 6. Zero inclusion regions for $n=10$ and $k=7$ with $a=0.4+0.4 i, a=0.3+0.2 i$, and $a=0.1+0.1 i$. 
of our inclusion regions for a trinomial with $n=10$ and $k=7$ when $a=0.4+0.4 i$, $a=0.3+0.2 i$, and $a=0.1+0.1 i$, respectively, where, as in the previous section, we just used the simple bounds from Lemma 2.6. The zeros are not indicated to avoid obscuring the small inclusion regions.

We conclude by mentioning that the same techniques we used here for trinomials could also be applied to quadrinomials of the form $z^{n}-a z^{k}-b z^{\ell}-1$ and other lacunary polynomials. However, key quantities playing a role similar to, for example, $\sigma(n, k)$ would in those cases have to be computed numerically, and the computations would generally become much more involved-well beyond the scope of the present work.

\section{Appendix}

Although the bounds in Lemma 2.6, which are explicit and require no extra computation, were sufficient for our purposes, better bounds lead to smaller inclusion regions. One may therefore sometimes want better approximations to the solutions of the real nonlinear equations we encountered before. Since that was not the focus of this work, we briefly describe efficient ways to accomplish this here.

We need approximations from above on the roots of $\chi_{k}$ and $\chi_{n-k}$ to compute approximations for the quantities $s$ and $t$ in Theorem 3.1, and approximations from above and below for the smallest and largest positive roots $r_{1}$ and $r_{2}$ of $\psi_{k}$, respectively.

First we consider

$$
\chi_{k}(x)=x^{n}-|a| x^{k}-1 .
$$

We set $y=y^{k}$ and compute approximations to the solution of $y^{n / k}-|a| y-1=0$. Since $n / k>1$, the function $y^{n / k}$ is convex, so the iterates generated by Newton's method from an initial point to the right of the root will all be upper bounds on that root. Such an initial point is easily obtained from Lemma 2.6 as $(1+|a|)^{k /(n-k)}$. Although Newton's method could also be used without the transformation $y=x^{k}$, convergence would be slower. The function $\chi_{n-k}$ can be treated analogously as $\chi_{k}$.

Now consider

$$
\psi_{k}(x)=x^{n}-|a| x^{k}+1 .
$$

Once again setting $y=x^{k}$ transforms the equation $\psi_{k}(x)=0$ into

$$
\psi_{k}\left(y^{1 / k}\right)=y^{n / k}-|a| y+1=0 .
$$

We need approximations that lie between the two positive roots of $\psi_{k}\left(y^{1 / k}\right)$. Although Lemma 2.6 provides an initial point, it is not possible to use any of the standard methods (Newton, Cauchy, Halley, et cetera) from such a point because 
the iterates may fall outside the interval determined by the roots. Instead, we approximate $y^{n / k}$ by a rational function of the form $\mu /(v-y)$, which coincides in function and derivative values with $y^{n / k}$ at a given point. This means that $\psi_{k}\left(y^{1 / k}\right)$ is approximated by

$$
\frac{\mu}{v-y}-|a| y+1
$$

We claim that the two roots of this approximation will lie between the roots of $\psi_{k}\left(y^{1 / k}\right)$. One can then continue with either one, depending on which root of $\psi_{k}\left(y^{1 / k}\right)$ one wishes to approximate. To verify the claim, we observe that if $\mu /(v-y)$ approximates $y^{n / k}$ to first order at a given point, then $(v-y) / \mu$, a linear function, must approximate $y^{-n / k}$ to first order, that is, it is its tangent at the given point. But $y^{-n / k}$ is a convex function, so $(v-y) / \mu \leq y^{-n / k}$, and therefore $\mu /(v-y) \geq y^{n / k}$. This means that the approximation (A-1) lies above $\psi_{k}\left(y^{1 / k}\right)$. Since the approximation is carried out at a point with a negative function value, the roots of the approximation must then lie between the roots of $\psi_{k}\left(y^{1 / k}\right)$ and will be closer to those roots than the point at which $\psi_{k}\left(y^{1 / k}\right)$ was approximated by the function in (A-1). The roots of the approximation are easily computed since, for the approximation at a point $\bar{y}$, a straightforward calculation shows that the values of $\mu$ and $\nu$ are given by

$$
\mu=\frac{k}{n} \bar{y}^{1+n / k} \quad \text { and } \quad v=\left(1+\frac{k}{n}\right) \bar{y} .
$$

Computing the roots of the function in (A-1) is equivalent to computing the roots of the quadratic equation

$$
|a| y^{2}-(1+|a| v) y+\mu+v=0 .
$$

Figure 7 illustrates the approximation (A-1) (dotted line) to $\psi_{k}\left(y^{1 / k}\right)$ (solid line) at the point where $\psi_{k}\left(y^{1 / k}\right)$ achieves its minimum.

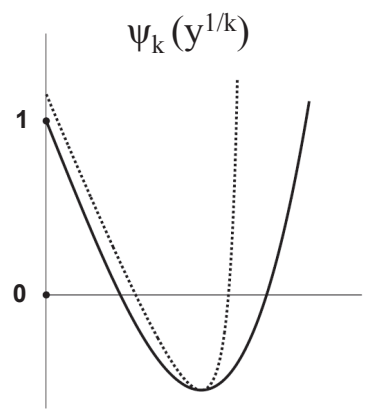

Figure 7. The function $\psi_{k}\left(y^{1 / k}\right)$ (solid line) and its approximation (dotted line). 


\section{References}

[Biernacki 1928] M. Biernacki, "Sur les équations algébriques contenant des paramètres arbitraires", Bulletin Acad. Polonaise 1927 (1928), 541-685. JFM 56.0863.02

[Cauchy 1829] A. L. Cauchy, Exercises de mathématique (1829), p. 122 in Oeuvres complètes, $2^{\mathrm{e}}$ série, vol. 9, Gauthier-Villars, Paris, 1891.

[Herglotz 1922] G. Herglotz, “Über die Wurzeln trinomischer Gleichungen”, Leipz. Ber. 74 (1922), 1-8. JFM 48.0082.01

[Kempner 1922] A. J. Kempner, "Über die Separation komplexer Wurzeln algebraischer Gleichungen”, Math. Ann. 85:1 (1922), 49-59. MR 1512044 Zbl 48.0088.03

[Kennedy 1940] E. C. Kennedy, "Bounds for the roots of a trinomial equation", Amer. Math. Monthly 47 (1940), 468-470. MR 2,116e Zbl 0060.05003

[Landau 1906] E. Landau, "Über den Picardschen Satz", Vierteljahrschrift Naturforsch. Gesellschaft Zürich 51 (1906), 252-318. JFM 57.1424.07

[Landau 1907] E. Landau, "Sur quelques généralisations du théorème de M. Picard", Ann. Sci. École Norm. Sup. (3) 24 (1907), 179-201. MR 1509080

[Lang 1999] S. Lang, Complex analysis, 4th ed., Graduate Texts in Mathematics 103, Springer, New York, 1999. MR 99i:30001 Zbl 0933.30001

[Marden 1966] M. Marden, Geometry of polynomials, 2nd ed., Mathematical Surveys 3, American Mathematical Society, Providence, R.I., 1966. MR 37 \#1562 Zbl 0162.37101

[Nekrassoff 1887] P. Nekrassoff, "Ueber trinomische Gleichungen”, Math. Ann. 29:3 (1887), 413 430. MR 1510423

[Rouché 1862] E. Rouché, "Mémoire sur la série de Lagrange", J. École Polytech. 22 (1862), 217218.

[Szabó 2010] P. G. Szabó, "On the roots of the trinomial equation”, CEJOR Cent. Eur. J. Oper. Res. 18:1 (2010), 97-104. MR 2011d:65110 Zbl 1204.65001

Received September 5, 2011. Revised April 17, 2012.

\section{AARON MELMAN}

DePARTMENT OF APPLIED MATHEMATICS, SCHOOL OF ENGINEERING

SANTA ClARA UNIVERSITY

SANTA Clara, CA 95053

UNITED STATES

amelman@scu.edu 


\title{
PACIFIC JOURNAL OF MATHEMATICS
}

\author{
http://pacificmath.org \\ Founded in 1951 by \\ E. F. Beckenbach (1906-1982) and F. Wolf (1904-1989)
}

\section{EDITORS}

V. S. Varadarajan (Managing Editor)

Department of Mathematics

University of California

Los Angeles, CA 90095-1555

pacific@math.ucla.edu

Vyjayanthi Chari

Department of Mathematics

University of California

Riverside, CA 92521-0135

chari@math.ucr.edu

\section{Robert Finn}

Department of Mathematics Stanford University

Stanford, CA 94305-2125

finn@math.stanford.edu

Kefeng Liu

Department of Mathematics

University of California

Los Angeles, CA 90095-1555

liu@math.ucla.edu
Darren Long

Department of Mathematics

University of California

Santa Barbara, CA 93106-3080

long@math.ucsb.edu

Jiang-Hua Lu

Department of Mathematics

The University of Hong Kong

Pokfulam Rd., Hong Kong jhlu@maths.hku.hk

Alexander Merkurjev

Department of Mathematics

University of California

Los Angeles, CA 90095-1555

merkurev@math.ucla.edu
Sorin Popa

Department of Mathematics University of California

Los Angeles, CA 90095-1555 popa@math.ucla.edu

Jie Qing

Department of Mathematics

University of California

Santa Cruz, CA 95064

qing@cats.ucsc.edu

Jonathan Rogawski

Department of Mathematics

University of California

Los Angeles, CA 90095-1555

jonr@math.ucla.edu

\section{PRODUCTION}

pacific@math.berkeley.edu

\section{SUPPORTING INSTITUTIONS}

ACADEMIA SINICA, TAIPEI

CALIFORNIA INST. OF TECHNOLOGY INST. DE MATEMÁTICA PURA E APLICADA KEIO UNIVERSITY

MATH. SCIENCES RESEARCH INSTITUTE NEW MEXICO STATE UNIV.

OREGON STATE UNIV.

\author{
STANFORD UNIVERSITY \\ UNIV. OF BRITISH COLUMBIA \\ UNIV. OF CALIFORNIA, BERKELEY \\ UNIV. OF CALIFORNIA, DAVIS \\ UNIV. OF CALIFORNIA, LOS ANGELES \\ UNIV. OF CALIFORNIA, RIVERSIDE \\ UNIV. OF CALIFORNIA, SAN DIEGO \\ UNIV. OF CALIF., SANTA BARBARA
}

\author{
UNIV. OF CALIF., SANTA CRUZ \\ UNIV. OF MONTANA \\ UNIV. OF OREGON \\ UNIV. OF SOUTHERN CALIFORNIA \\ UNIV. OF UTAH \\ UNIV. OF WASHINGTON \\ WASHINGTON STATE UNIVERSITY
}

These supporting institutions contribute to the cost of publication of this Journal, but they are not owners or publishers and have no responsibility for its contents or policies.

See inside back cover or pacificmath.org for submission instructions.

The subscription price for 2012 is US \$420/year for the electronic version, and \$485/year for print and electronic.

Subscriptions, requests for back issues from the last three years and changes of subscribers address should be sent to Pacific Journal of Mathematics, P.O. Box 4163, Berkeley, CA 94704-0163, U.S.A. Prior back issues are obtainable from Periodicals Service Company, 11 Main Street, Germantown, NY 12526-5635. The Pacific Journal of Mathematics is indexed by Mathematical Reviews, Zentralblatt MATH, PASCAL CNRS Index, Referativnyi Zhurnal, Current Mathematical Publications and the Science Citation Index.

The Pacific Journal of Mathematics (ISSN 0030-8730) at the University of California, c/o Department of Mathematics, 969 Evans Hall, Berkeley, CA 94720-3840, is published monthly except July and August. Periodical rate postage paid at Berkeley, CA 94704, and additional mailing offices. POSTMASTER: send address changes to Pacific Journal of Mathematics, P.O. Box 4163, Berkeley, CA 94704-0163.

PJM peer review and production are managed by EditFLOW ${ }^{\mathrm{TM}}$ from Mathematical Sciences Publishers.

PUBLISHED BY PACIFIC JOURNAL OF MATHEMATICS

at the University of California, Berkeley 94720-3840

A NON-PROFIT CORPORATION

Typeset in LATEX

Copyright $(02012$ by Pacific Journal of Mathematics 


\title{
PACIFIC JOURNAL OF MATHEMATICS
}

\author{
Volume $259 \quad$ No. $1 \quad$ September 2012
}

Extension Theorems for external cusps with minimal regularity

GABRIEL ACOSTA and IGNACIO OJEA

Convergence of axially symmetric volume-preserving mean curvature flow

MARIA ATHANASSENAS and SEVVANDi KANDANAARACHCHI

On the horoboundary and the geometry of rays of negatively curved manifolds

FranÇOISE DAL'bo, Marc PEIGNÉ and ANDREA

SAMBUSETTI

Two infinite versions of the nonlinear Dvoretzky theorem

KEI FUNANO

Nonlocal uniform algebras on three-manifolds

ALEXANDER J. IZZO

Mahlo cardinals and the torsion product of primary abelian groups

PATRICK W. KEEF

Geometry of trinomials

AARON MELMAN

Drinfeld orbifold algebras

ANNE V. SHEPLER and SARAH Witherspoon

Semi-topological cycle theory I

JYH-HAUR TEH

New construction of fundamental domains for certain Mostow groups 209

TiEHONG ZHAO 